\title{
Die Schulsituation in der Pandemie aus Sicht der Landesschüler*innenvertretung NRW
}

unter Mitarbeit von Julius van der Burg und Pia Sophie Kogler

Dieser Beitrag beschäftigt sich damit, was in Schulen während der Pandemie aus Schüler*innenperspektive falsch gelaufen ist. Die Inhalte des Beitrags beruhen auf der Arbeit der Landesschüler*innenvertretung NRW, welche in regem Austausch zu Schüler*innen steht und deren Interessen vertritt. Dabei liegt der Fokus auf der dramatischen Chancenungleichheit des Home-Schoolings und es werden mögliche, langfristige Konsequenzen dargestellt. Des Weiteren wird ein Ausblick gegeben mit konkreten Vorschlägen, wie das Schulsystem krisensicher und gerecht(er) werden kann.

Nicht ohne Grund war Schule während der Pandemie eines der größten Themen in den Medien. Die Pandemie zeigte auf, dass Schule weder krisensicher ist, noch digitalisiert und modern. Die Probleme, die es im Schulsystem schon lange gibt, wie die fehlende Digitalisierung, veraltete Gebäude und den Lehrerinnenmangel, wurden durch die Pandemie nun gesehen.

Diese Probleme sind aber so tiefgreifend, dass sie nicht innerhalb von Monaten oder Jahren lösbar sind. Gerade in einer Pandemie ist es nicht möglich, die Probleme in ihrem Kern zu lösen. Stattdessen werden nur die Folgen bekämpft mit eingeschränktem Erfolg. Dass Schule nie eine wirkliche Priorität der Landesregierung war, rächt sich in der Pandemie. So besagt eine Studie, dass im ersten Lockdown Schülerinnen genau so viel gelernt hätten wie in den Ferien - gar nichts. Dies unterstreicht die Situation von Schule in der Pandemie.

Schule in der Pandemie ist gut vergleichbar mit einem Kind, das gerade lernt zu schwimmen. Es kann sich über Wasser halten, mehr aber auch nicht. So waren die Lehrkräfte überfordert, es fehlte an Konzepten und die Landesregierung arbeitete mit einem Fokus auf Leistung und Abschlüssen, anstatt die Gesundheit der Schüler^innen zu priorisieren. Abschlüsse wurden standardisiert geschrieben und Schüler`innen weiterhin bewertet. Während die Welt in einer Ausnahmesituation ist, und damit Schule auch, ändert sich nichts am Bewertungssystem. Dies ist aus der Sicht der Schüler^innen einer der fatalsten Fehler des Krisenmanagements in Bezug auf Schule. 
Auch Chancenungleichheit ist eines der großen Themen. Home-Schooling war ein Privileg und für Schüler^innen aus sozialschwächeren Familien nur ein Euphemismus. Vor allem frustrierend war auch, dass gegen diese Umstände nichts getan wurde. Bis auf die Anschaffung von digitalen Endgeräten gab es keine Maßnahmen, welche die Chancen für Schüler`innen aus sozialschwächeren Familien auf gute Bildung bessern.

Auch die Schülerinnenvertretungsarbeit während der Pandemie war vor allem frustrierend. Die Präsenz in Medien und der rege Austausch mit der Politik sorgten zwar dafür, dass die Popularität von Schüler`innenvetretungsverbänden stieg, änderte aber nichts an dem politischen Diskurs und den Maßnahmen an Schulen. Die Meinung der Schüler^innen wurde gehört, aber nicht ernst genommen. Genau deshalb war Schule in der Pandemie so negativ für Schülerinnen: Die Wünsche wurden abgetan unter dem Motto, Politik und Erwachsene wüssten besser, was die Schüler`innen brauchen. Diese Annahme sorgte dafür, dass Schule von Erwachsenen für Schüler*innen organisiert wurde, anstatt die Betroffenen in den Entscheidungsprozess einzubeziehen.

Zusammenfassend lässt sich sagen, dass Schule in der Pandemie nicht funktioniert hat. Es hat sich gezeigt, dass man das Schulsystem neu denken muss und Schüler*innen ernst nehmen sollte, wenn man zu ihren Gunsten Entscheidungen treffen will. Bis jetzt scheint es nicht, als hätte die Politik aus ihren Fehlern gelernt. Trotzdem kann diese Pandemie ein Sprungbrett sein, um Schule krisensicher, modern und gerechter zu gestalten. In Schulen ist in den ersten 18 Monaten der Pandemie viel falsch gelaufen und es braucht nun kurzfristige Maßnahmen und langfristige Konzepte, um Corona zu verarbeiten und Schule krisensicher zu machen.

\section{Welche Themen waren in den letzten Jahren für die Schüler*innen besonders brennend?}

Die Pandemie hat vor allem die Schule und das Schulsystem vor besondere Herausforderungen gestellt. Diesen musste sich die Schule als System stellen, aber auch alle Schüler`innen standen vor besonderen Herausforderungen, die den Schulalltag und das Leben in der Schule stark verändert haben. Deswegen gab es in der Coronazeit einige Themen, die das Schulleben der Schüler*innen bestimmt haben. Ein großes Thema war die psychische Gesundheit.

Die Zeit des Home-Schoolings bedeutete für viele Schüler*innen, dass der Leistungsdruck sich verstärkte, der im deutschen Schulsystem (auch außerhalb der Coronapandemie) den Schulalltag bestimmt. Auch der soziale Ausgleich, der 
normalerweise durch Treffen mit Freund`innen möglich, war durch die notwendigen Corona-Beschränkungen, nicht mehr gegeben. In Verbindung mit der Angst vor einem schweren COVID-Verlauf oder der Angst um Familienmitglieder und Freunde sorgte dies bei vielen Schüler*innen dafür, dass sie dem Unterrichtsstoff nur schwer oder gar nicht mehr folgen konnten und die seelische Gesundheit bei vielen stark litt.

Hinzu kam, dass Fälle häuslicher Gewalt zugenommen haben. Kontrollmechanismen, wie zum Beispiel ein ${ }^{\star}$ Klassenlehrer`in, die Verletzungen und blaue Flecken bemerken kann, gab es durch den Distanzunterricht im Home-Schooling nicht mehr, auch Vernachlässigung im häuslichen Kontext konnten nicht mehr durch die Schule bemerkt und aufgefangen werden.

Auch die Benotung des Home-Schoolings führte zu einigen Problemen. Für Lehrer*innen war es häufig nur schwer möglich, den individuellen Lernstand und Leistungsstand einzelner Schüler`innen zu ermitteln. Dadurch war auch die Notengebung schwer bis unmöglich. Dazu kommt, dass im Home-Schooling der sozioökonomische Hintergrund der Familie des`r einzelnen Schüler^in noch entscheidender für den individuellen Bildungserfolg wurde. In einigen Familien konnte beim Erklären von Themen geholfen werden, die technische Ausstattung war gegeben, um am Distanzunterricht teilzunehmen, oder es gab eine ruhige Lernumgebung, wie zum Beispiel einen eigenen Raum, in dem nicht noch andere Menschen arbeiteten. Dies alles sorgt dafür, dass sich die erbrachten Leistungen drastisch verschieben, da es nicht nur auf das erbrachte Engagement und die Motivation ankommt, sondern in erster Linie auf die sozioökonomischen Möglichkeiten der Familie.

Es wurden zwar einige Maßnahmen ergriffen, um die beschriebenen Probleme zu bekämpfen, wie zum Beispiel das Verteilen von mobilen Endgeräten und das Einrichten von Study-Halls, also Räumen, aus denen Schüler*innen am Distanzunterricht teilnehmen konnten. Diese Maßnahmen waren zwar wichtig, aber nicht weitreichend genug, um die sozialen Ungerechtigkeiten, die durch die Coronapandemie aufkommen, zu bekämpfen. Wenn in den kommenden Jahren nicht darauf geachtet werden wird, die Schüler*innen aufzufangen, die durch die Coronapandemie und ihre häuslichen Umstände abgehängt wurden, wird die Pandemie die soziale Gerechtigkeit und die Aufstiegsmöglichkeiten für viele Menschen stark zurückwerfen.

Die Schulabschlüsse, wie zum Beispiel das Abitur, waren ein weiteres Thema, das viele Schüler*innen stark beschäftigte. So mussten Schülerinnen ein zentrales Abitur schreiben, das zwar durch kleine Anpassungen der besonderen Situation gerecht werden sollte, dies aber nicht konnte. Das Abitur, das auch außerhalb von der Coronapandemie kaum Vergleichbarkeit schafft, schaffte in diesem Jahr noch weniger Vergleichbarkeit, weil die Situation der Schüler*innen von 
Schule zu Schule und von Elternhaus zu Elternhaus stark variierte. Einige Schüler*innen hatten mehr Präsenzunterricht als andere und auch der Distanzunterricht funktionierte von Schule zu Schule unterschiedlich gut. Dies führte dazu, dass die Möglichkeiten zur Vorbereitung auf Abschlussprüfungen im Präsenzunterricht von Prüfling zu Prüfling sehr unterschiedlich waren.

\section{Welche Rolle kam der LSV zu?}

Die Landesschüler^innenvertretung (LSV) NRW ist die Interessenvertretung aller etwa 2,6 Millionen Schüler^innen Nordrhein-Westfalens. Vor Corona fokussierte sich die Arbeit der LSV NRW vor allem auf eine langfristige Neugestaltung von Schule und forderte bspw. die Einführung der Inklusiven GanztagsGesamtSchule (IGGS). Während der Pandemie rückte Schule, und damit auch Schüler*innenvertretungsarbeit, in den Fokus. Die LSV NRW war präsenter in den Medien und konnte den Grad an Bekanntheit signifikant steigern. Dadurch erreichten den Landesvorstand der LSV NRW viele Anfragen und Mails, in welchen Schüler*innen ihre Sorgen, Wünsche und Frust verdeutlichten. So herrscht seit März 2020 enger Kontakt mit Schüler`innen, welche an die LSV NRW herantreten. Die LSV NRW versteht sich als Gemeinschaft und Lobby für die Schüler*innen NRWs und unterstützte und beriet jede ${ }^{\star} n$, welche ${ }^{\star} r$ sich meldete. Des Weiteren etablierte die LSV monatliche Angebote des Austauschs und startete Social-Media-Kampagnen, um mit den Schüler^innen in Kontakt zu treten. Zusammenfassend entwickelte sich die LSV NRW zu einem Anlaufpunkt für alle besorgten Schüler^innen und vereinigte deren Sorgen in einer lauten Stimme gegen das Krisenmanagement der Landesregierung.

Darüber hinaus wurde die Rolle der LSV als Interessenvertreter der Schüler*innen immer wichtiger. Schule und die immensen Einschränkungen im Schulalltag rückten in den Fokus der Medien. Schüler`innen schienen interessant dadurch nahm die LSV schnell die Rolle der repräsentativen Stimme in Politik und Medien ein. Pressemitteilungen, Pressetermine und der Austausch mit Politiker*innen dominieren seit März 2020 die Arbeit der LSV. Dadurch rückte die LSV von ihrer Arbeit ab, welche sich mit langfristigen Problemen auseinandersetzte, und wurde deutlich tagesaktueller. Die LSV gewann an Popularität bei Schüler^innen, Eltern, Lehrerinnen und Politiker`innen und konnte im Austausch mit Verbänden und dem Ministerium für Schule und Bildung die Schüler^innen effektiver vertreten. Daraus resultierte, dass die LSV nicht nur in den Medien ihre politische Stimme für die Schüler`innen stärken konnte, sondern sich auch die Politiker^innen durch den öffentlichen Druck mit den Positionen und Mei- 
nungen der Schüler*innen NRWs auseinandersetzen mussten. So konnte die LSV kleine Erfolge während der Pandemie verzeichnen, wie bspw. das Wegfallen der Abweichprüfungen bei verbesserten Noten.

Diese Arbeit und die neuen Funktionen der LSV sind weiterhin aktuell und geben eine hoffnungsvolle Perspektive: Auch nach der Pandemie gibt es in der Schule Probleme, die von der Pandemie aufgezeigt wurden. Schüler`innen dürfen jetzt nicht von der Bildfläche verschwinden, sondern müssen im politischen Diskurs weiterhin präsent bleiben. Die LSV möchte ihre Rolle als öffentlicher Vertreter deshalb beibehalten und die Pandemie als Sprungbrett nutzen, um Bildung und Schule endlich in der politischen Debatte zu priorisieren.

\section{Welche Themen wurden von Mitschüler*innen an uns herangetragen?}

Mit der zunehmenden Popularität der LSV NRW wuchs auch die Anzahl an Mails, welche von Schüler^innen aus ganz NRW geschrieben wurden. Dabei wurden nicht nur die verschiedensten Schicksale während des Lockdowns beschrieben, sondern auch diverse Sorgen und Befürchtungen geteilt.

Vor allem während des Home-Schoolings teilten Schüler^innen ihre Ängste darüber, wie der neue Lernort, oft das eigene Kinderzimmer, ihre Leistungen beeinflussen wird. Da Distanzunterricht von Lehrkräften seit August 2020 bewertet wurde, standen die Schüler^innen im Home-Schooling unter extremem Druck sie mussten eigenständig und alleine lernen unter den unterschiedlichsten Voraussetzungen. So gibt es Schüler*innen, welche von einem starken Leistungsabfall, fehlendem Onlineunterricht und nur Langzeitaufgaben berichten, während andere Schulen das Konzept Digitalunterricht schnell und gut umsetzen konnten. Diese Ungleichheit bestärkt für Schüler*innen die Absurdität einer Bewertung des Home-Schoolings. Viele fragten frustriert, in welcher Welt es fair sei, dass ein ${ }^{\star}$ S Schüler ${ }^{\star}$ in, welche ${ }^{\star}$ r zu Hause auf drei Geschwisterkinder aufpassen muss, auf der gleichen Grundlage bewertet wird wie das Einzelkind, das während des gesamten Lockdowns Nachhilfestunden hatte? Die Frage nach der Gerechtigkeit und dem Sinn hinter einer starren Bewertung des Distanzunterrichtes ist nun aktueller denn je, denn die Lernlücken, welche durch die Lockdowns entstanden sind, führen nun dazu, dass Schüler*innen im Präsenzunterricht abgehängt sind. Diese Schüler*innen stehen vor der Herausforderung, Unterrichtsinhalte von etwa fünf Monaten Home-Schooling allein aufzuarbeiten, zu wiederholen und zu riskieren, dass der angestrebte Schulabschluss im Endeffekt nur ein Traum bleibt. „Es wird erwartet, dass wir uns an die Pandemie anpassen, und nicht die Pande- 
mie an uns", fasst der Landesvorstand in einer Pressemitteilung zu den Aufholangeboten während der Sommerferien 2021 die Sorgen und das große Problem der Schüler`innen zusammen.

Ein weiterer großer Kritikpunkt der Schüler`innen waren die fortlaufenden Klausuren und Abschlussprüfungen. Das Ministerium für Schule und Bildung NRW entschied sich früh dagegen, jegliche Inhalte für die nächsten Abschlussjahrgänge anzupassen, weiterhin wurde nach den standardisierten Lehrplänen unterrichtet. Daraus resultierte auch, dass Klausuren und Abschlussjahrgänge bis auf kleinste Veränderungen wie immer geschrieben wurden. Auch dies hielten und halten die Schüler`innen NRWs für ungerecht und befürchten, dass die Inhalte in Prüfungen zu schwer seien - schließlich sorgte der Distanzunterricht dafür, dass viele Kurse und Klassen hinter den Lehrplänen zurückblieben. Die Befürchtungen der Schüler^innen bewahrheiteten sich letztendlich auch: Die Abiturklausuren in den Fächern Mathematik und Englisch wurden stark kritisiert. Viele Schüler`innen teilten ihre Wut darüber, dass das Ministerium entgegen der eigenen Ankündigung handelte, faire, und an die Pandemie angepasste Klausuren zu stellen.

Darüber hinaus stellt das Hin- und Her in Entscheidungen und Schulöffnungen ein großes Problem für die Schülerinnen da, welche bemängeln, dass sie keine Sicherheit verspüren und deshalb unsicher in ihren Schulalltag hineinleben. Dies ist in einer Zeit, welche sowieso von Unsicherheit geprägt ist, eine besondere Belastung, denn Schule ist für Schüler*innen das stabile Standbein ihres Lebens. Diese Dynamik in Entscheidungen zieht sich mittlerweile durch die ganze Pandemie und wird seit jeher stark kritisiert.

Aus den genannten Erfahrungen entwickelte sich der Eindruck, dass Noten und Abschlüsse im Krisenmanagement des Schulministeriums oberste Priorität haben. So erzählen viele Schüler`innen, dass ihre seelische Gesundheit stark unter dem Leistungsdruck, der Isolation und der sozialen Verarmung litt und leidet. Anstatt die ganze Zeit Klausuren zu schreiben, hätten sie viel eher ein Angebot gebraucht, dass die Zeit im Home-Schooling verarbeitet - zusammen mit Schulsozialarbeiter`innen und Schulpsycholog*innen. Trotz diesen Feedbacks, welches auch von Expert*innen unterstrichen wird, wurde auch nach der Rückkehr in den Präsenzunterricht am 31.05.2021 der Fokus auf Klassenarbeiten gesetzt, egal ob in Klasse 5 oder 12. Dies sorgte dafür, dass die seelische Belastung der Schüler`innen sich weiter intensivierte - die Vorfreude auf die Freunde war gedämpft und der Fokus lag auf den Leistungen. Dies berichten nicht nur Schüler*innen, sondern auch besorgte Eltern, welche appellieren, dass Schule in besonderen Zeiten eben auch besonders, und nicht standardisiert, gedacht werden müsse. 


\section{Langfristige Folgen}

All diese Herausforderungen in der Pandemie werden vermutlich auch langfristig Schule und Schüler^innen beeinflussen. Auch wenn die Pandemie vorbeigeht, bleiben die Lernlücken. In Bezug auf diese Lernlücken muss auch darüber nachgedacht werden, was die Pandemie für die Chancengleichheit bedeutet hat.

Home-Schooling war für viele Schüler*innen ein Euphemismus und keine Realität. Die Ungleichheit, die in Schulen aufgrund von sozialer Herkunft sowieso herrscht, wurde durch die Pandemie befeuert. So saßen Schüler*innen ohne Unterstützung und mit veralteten Geräten zu Hause und verloren den Anschluss an den Unterricht. Dadurch entstanden bei diesen Kindern größere Lernlücken, welche ohne Tutor*innen oder individuelle Unterstützung nicht mehr aufzuarbeiten sind. Diese Angebote sind aber meist durch finanzielle Mittel zu erwerben Mittel, die gerade Schülerinnen aus sozial schwächeren Schichten nicht haben. Das bedeutet, dass diese Schüler*innen in den Präsenzunterricht starten mit Lernlücken und ohne Aussicht auf Unterstützung. Dies führt zu schlechten Noten und kann langfristig auch über den Schulabschluss entscheiden. Ein Schulabschluss bestimmt im Endeffekt auch die Möglichkeiten, die ein Mensch im Leben hat. Daher befürchtet die LSV NRW, dass durch die Pandemie die Vererbung von sozialen Ungleichheiten noch intensiver wird, da nicht-privilegierte Schüler^innen im Home-Schooling alleine gelassen wurden und ihrer Situation ausgeliefert waren.

Erschwerend kommt hinzu, dass auch der Distanzunterricht seit dem Schuljahr 2020/2021 bewertet wird. Das bedeutet, dass Schüler*innen neben den finanziellen Sorgen in ihrer Familie und fehlenden digitalen Geräten trotzdem einen Weg finden mussten, Leistung zu zeigen. Aus Nachrichten an die LSV NRW geht hervor, dass den Schüler`innen währenddessen durchaus bewusst war, in was für einer ungerechten Lage sie sich befinden. So saßen einige Mitschüler^innen mit privaten Tutor ${ }^{\star}$ innen und guter technischer Ausstattung zu Hause, während andere auf vier Geschwister aufpassen mussten und sich mit diesen auch ein Gerät teilten. Obwohl also zu Hause keine Vergleichbarkeit zum Bewerten für die Lehrkraft vorlag, wurden Schüler*innen allein anhand ihrer schulischen Leistung bewertet. Diese Ungerechtigkeit frustrierte vor allem jene Schüler`innen, welche zu Hause schlechtere Bedingungen hatten. Durch Corona selektiert Schule also passiv nach sozialer Herkunft und bestimmt kurzfristig Noten und langfristig Lebensrealitäten von Schüler*innen.

Dies betrifft auch die Jüngsten. In einem WDR5 Tagesgespräch erzählte ein besorgter Vater die Geschichte seiner 9-jährigen Tochter. Die Familie waren Immigranten und dadurch war es den Eltern nicht möglich, ihrer Tochter schuli- 
sche Unterstützung während des Home-Schoolings zu geben. Nun befürchtet er, dass seine Tochter doch keine Gymnasialempfehlung bekommen und stattdessen auf eine Realschule gehen werde. Corona und die Bedingungen zu Hause seien die einzigen Gründe dafür, dass sein Kind schlechtere Chancen auf einen hohen Bildungsweg bekomme. Dieses Beispiel ist nur ein Schicksal von vielen, welche durch die Pandemie langfristig negativ beeinflusst werden. So gab es allein im ersten Corona-Schuljahr doppelt so viele Schulabbrecherinnen, wie in dem Jahr zuvor.

Dies ist alarmierend und zeigt, dass Schule jetzt andere Prioritäten braucht, als alle Inhalte des Lehrplans zu erarbeiten. Dabei darf auch nicht vergessen werden, dass die Schüler`innen unter der Pandemie seelisch gelitten haben. Psycholog*innen warnen schon lange vor den Folgen, die die Pandemie auf die seelische Gesundheit von jungen Menschen haben wird. Der Leistungsdruck in der Schule bestärkte neben der Isolation den Druck und die Verzweiflung der jungen Menschen. Wenn Schule jetzt wie gewohnt weitermacht und nur Leistung anstatt den Menschen sieht, dann ist zu befürchten, dass noch mehr Schüler*innen sich in dem Druck verlieren und sich alleine gelassen fühlen. Deshalb braucht es nun Angebote an Schulen, um die Erfahrungen mit der Pandemie zu verarbeiten. Darüber hinaus müssen die Lerninhalte auf die Pandemie und die Zeit im Home-Schooling angepasst werden, anstatt Inhalte unter Zeitstress und ohne wirkliche Zeit zum Erklären zu vermitteln. „Das seelische Wohl der Schüler`innen muss wichtiger sein, als das Ablegen von Prüfungen oder das standardisierte Bewerten!“, betonte die LSV NRW in einer Pressemitteilung zusammenfassend.

\section{Jugendpartizipation - Mitbestimmung während der Pandemie}

Die Coronapandemie hat unser aller Leben maßgeblich beeinflusst und viele ungewohnte Dinge ganz alltäglich gemacht. Wer vor ein paar Jahren prophezeit hätte, dass wir demnächst alle mit Masken herumlaufen würden und Jens Spahn auf einmal fast relevanter ist, als die Bundeskanzlerin, wäre damals für verrückt erklärt worden. Doch die Pandemie hat genau dazu geführt.

Aber auch im Bereich Schule entstanden viele Veränderungen in diesen besonderen Zeiten. Dabei wurde eins schon zu Beginn der Pandemie klar: Bei all diesen Veränderungen, die aus gesundheitlicher Sicht notwendig waren, würde die Landesregierung dieselben Fehler begehen, wie sie es seit 2017 schon tut. Durch die Maßnahmen wurde das Leben der Schülerinnen massiv verändert. Natürlich ist das bei allen Bevölkerungsschichten der Fall gewesen, Schüler`innen sind da- 
bei aber, in ihrem zur Gesamtbevölkerung verhältnismäßig jungen Alter, viel mehr auf soziale Kontakte und auch die verschiedenen Freiheiten angewiesen, als es andere sind.

In einer Zeit, in der Kinder Grundlagen der sozialen Teilhabe an der Gesellschaft erleben, in der Jugendliche die Autonomie vom Elternhaus anstreben und in der die wichtigsten sozialen Grundlagen für das zukünftige Leben entstehen, ist Isolation das Destruktivste, was in dieser Situation passieren kann. Wenn man dann Kontakte reduzieren muss, wie es in der Coronapandemie unstrittig der Fall war, ist es notwendig, dies im Abwägen zwischen medizinischer Gesundheit und sozialen Komponenten zu tun. Es muss demnach natürlich überlegt werden, welche Einschränkungen, unter Berücksichtigung dieser Faktoren, sinnvoll sind.

Und nicht nur oben Genanntes muss in seiner Sinnhaftigkeit kontrovers diskutiert werden, sondern auch andere Maßnahmen. Aus schulpolitischer Sicht heißt das beispielsweise, wie man mit einer durch die Pandemie verstärkten starken Chancenungleichheit umgeht, wie Unterricht auf Distanz am besten auszusehen hat und wie Schüler*innen in dieser schwierigen Zeit entlastet werden können, anstatt sie durch den enormen Leistungsdruck weiter in Existenzsorgen $\mathrm{zu}$ treiben.

Um einen solchen Austausch aber zu erreichen, braucht es vor allem eins die Meinung der wirklich Betroffenen. Wie soll man auch Maßnahmen beschließen, wenn man selbst zuletzt vor 30 Jahren eine Schule von innen gesehen hat und keinen Vergleichswert hat, welche Maßnahmen welche Wirkung erzielen? Immerhin gehen vermutlich die meisten Politiker*innen einfach davon aus, dass mit mehreren Milliarden Euro für digitale Endgeräte alle Probleme der Schulen gelöst sind, ohne die eigentliche Realität in Schulen zu kennen. Realität ist nämlich, dass von solchen Geldern kaum etwas die Schulen überhaupt erreicht. Die Probleme von Schule sitzen viel tiefer als nur in der schlechten Ausstattung und dem hohen Investitionsstau, der am Ende nur eines der vielen Puzzleteile für eine gerechte Bildung und eine lebenswerte Schule ist. Wie auch, wenn am Ende für die Entscheidung schlichtweg die Erfahrungsberichte fehlen, die nur durch einen pluralistischen Austausch erlangt werden können?

Dennoch davon auszugehen, dass man ohne diesen Bezug schulpolitische Angelegenheiten lösen kann, ist der ständige Irrglaube der schwarz-gelben Landesregierung - der ständige Fehler, wie sie ihn schon seit 2017 begeht. Es benötigt also einen zivilgesellschaftlichen Prozess, um durchzusetzen, dass politische Entscheidungen immer unter Anhörung von Betroffenen besprochen werden müssen, die die Maßnahmen wirklich erleben und die aus der Praxis über den realen Alltag berichten können.

Im Falle der Schule braucht es dafür vor allem Schüler*innen, die aktiv in die Entscheidungsfindung eingebunden werden. Erst wenn Schüler*innenvertre- 
tungen auf kommunaler, Bezirks- und Landesebene mit verbindlichem Mitbestimmungsrecht in die politischen Gremien aufgenommen werden, kann sich die Schule nachhaltig und progressiv entwickeln. Das Bedürfnis der Schüler`innen für eine nachhaltige Partizipation sieht man spätestens seit ein paar Jahren durch die FridaysForFuture-Bewegung und viele weitere Gruppen aus der Klimagerechtigkeitsbewegung.

Auch wir Schüler*innen versuchen uns immer wieder bestmöglich in politische Entscheidungen einzubringen. Gerade durch die Coronapandemie war es fast unumgänglich für viele Schüler*innen, mit der politischen Arbeit anzufangen, da viele Maßnahmen katastrophale Auswirkungen auf den Schulalltag hatten. Gegen solche Maßnahmen wollten und wollen sich viele Schülerinnen einbringen, um progressivere Regelungen umzusetzen. Immerhin ist diese Art des Engagements für viele die einzige Möglichkeit, ihre Ideen in die Politik zu tragen, wenn man nicht einmal wählen darf, um die Regierung mitzubestimmen. Doch gerade in solchen Situationen schafft es die Regierung immer wieder, gerade kritische Stimmen zu überhören und Schüler`innen aus dem politischen Diskurs herauszuhalten.

\section{Was wünschen wir uns von der Politik und der Gesellschaft?}

Schule existiert nicht in einem luftleeren Raum. Sie ist ein Spiegel der Gesellschaft und muss als solcher betrachtete werden. Deswegen können wirkliche Veränderungen nur erreicht werden, wenn wir uns als Gesellschaft den großen Problemen annehmen, die in der Schule sichtbar werden und uns wirklich die Frage stellen, was sich gesamtgesellschaftlich verändern muss, damit Schule mehr als nur ein Ort wird, an dem Schüler*innen Wissen eingetrichtert wird, ein Ort des gemeinsamen Lernens, der gemeinsamen Entwicklung und des gemeinsamen Lebens.

Im Moment jedoch ist Schule ein Ort, an dem ein enormer Leistungsdruck herrscht. Schule soll Schüler*innen an die spätere Arbeit gewöhnen und an den Druck, der im Berufsleben herrscht. Deswegen müssen sich Dinge wie seelische Gesundheit und die Entwicklung des einzelnen häufig hintenanstellen. Auch in der Pandemie ließ sich beobachten, dass körperliche und seelische Gesundheit sich der Benotbarkeit unterordnen mussten. So wurde zum Beispiel das Abitur mit nur kleinen Veränderungen geschrieben, während viele Schülerinnen gerade stark unter psychischen Problemen litten und große Angst vor Erkrankungen hatten. 
Das Problem mit Benotungen startet aber eigentlich schon viel früher. Noten geben den Eindruck, als seien sie objektiv und würden für Vergleichbarkeit sorgen. Dieses Gefühl ist aber falsch. So hört man immer wieder davon, dass zum Beispiel NRW ein leichteres Abitur habe als Bayern, aber die Probleme mit der Vergleichbarkeit gehen noch tiefer: So kann es schon einen großen Unterschied machen, an welcher Schule man unterrichtet wird, weil der Stand der technischen Ausstattung sehr unterschiedlich sein kann. Schon die Frage, bei welcher Lehrperson man unterrichtet wird, kann einen großen Einfluss auf die Benotung haben. Deswegen fordert die LSV NRW die Abschaffung der Noten, sodass die Entwicklung von Schüler*innen wieder Vorrang hat vor dem Versuch, eine gute Note - die kaum eine Aussagekraft über die jeweiligen Schülerinnen und Schüler hat - zu bekommen. Außerdem muss die Gesellschaft ihre Wahrnehmung von Noten verändern. Menschen sind viel mehr als ihre Noten. Das ständige Achten auf die Noten verstärkt den Leistungsdruck enorm und sorgt bei einigen Schüler`innen für große Probleme mit der seelischen Gesundheit.

Darüber hinaus muss Schule ein Ort werden, an dem alle Schüler*innen zusammen leben und lernen können. Dafür muss die Mehrgliedrigkeit des Schulsystems aufgehoben werden. Häufig wird schon nach der vierten Klasse die Zukunft des*der Einzelnen bestimmt. Dabei sind die sozioökonomischen Hintergründe der Schüler*innen entscheidender als die Leistungen, die man tatsächlich erbringen kann. Zudem verändern sich Schülerinnen nach der vierten Klasse noch stark, sodass eine Einteilung viele Chancen verdirbt. Ein Ort an dem alle zusammen lernen beinhaltet aber auch Menschen mit Behinderung, die in unserem Schulsystem auf Förderschulen kommen, auf denen nur Menschen mit Behinderungen sind. Es braucht aber einen Ort des gemeinsamen Lernens, bei dem niemand aufgrund seiner Herkunft, seiner Hautfarbe oder wegen einer Behinderung diskriminiert wird. Damit die Schule aber ein solcher Ort werden kann, muss die Gesellschaft die Exklusion von Menschen mit Behinderung stoppen. Menschen mit Behinderung müssen an der Gesellschaft teilnehmen können und alle Chancen haben können, die Menschen ohne Behinderung auch haben. Dafür muss mit einem Wirtschaftssystem gebrochen werden, das Menschen aufgrund ihrer Leistungsfähigkeit diskriminiert. Es braucht also eine Inklusive-GanztagsGesamtschule, in der Schüler^innen zusammen lernen und leben können und in der niemand aufgrund dessen, was er*sie leisten kann, diskriminiert wird. Dafür braucht es eine Gesellschaft, in der jede ${ }^{\star}$ r teilhaben kann, egal, wie der sozioökonomische Hintergrund aussieht, oder ob Menschen eine Behinderung haben oder nicht.

Zudem müssen Schüler*innen eine feste Rolle in den politischen Entscheidungsprozessen bekommen. Es muss feste Partizipationswege geben, die Gehör in der Politik finden und es braucht ein Demonstrationsrecht für Schüler`innen 
während der Schulzeit, sodass Schüler^innen sich Gehör verschaffen können. Wie soll sonst eine Schule entstehen, die das Beste für Schüler^innen will, wenn die Betroffenen nicht einmal angehört werden?

Um die Folgen der Coronapandemie abzumildern, braucht es kurzfristig eine Dezentralisierung der Abschlüsse sowie eine Anpassung der Lehrinhalte. Auch muss es neue Konzepte für Home-Schooling geben wie bspw. kostenlose Tutor^innen für Schüler^innen, sowie das Aussetzen der Bewertung im Distanzunterricht. Darüber hinaus müssen Schulpsycholog^innen sowie Schulsozialarbeiter^innen eingestellt werden, welche mit den Schüler`innen die Pandemie verarbeiten. Die Schulpolitik muss jetzt zeigen, dass die Schüler^innen als Menschen wichtiger sind als gute Noten und standardisiertes Bewerten. Langfristig aber braucht es die oben genannten Maßnahmen, um Schule krisensicher und gerechter zu gestalten.

Zusammenfassend bedarf es einer an ihre Wurzel gehende Umstrukturierung unserer Gesellschaft. Denn eine Schule, die dem Leistungsdruck und der kapitalistischen Logik unterworfen ist, wird allerhöchstens Menschen erzeugen, die großen Druck gewohnt sind - und nicht selbsterfüllte Individuen, die zur Teilhabe in der Gesellschaft erzogen wurden. 